\title{
Stokastik İlişkisiz Paralel Makine Çizelgeleme Problemi için bir Matematiksel Model
}

\author{
Mehmet Ertem ${ }^{1 *}$, Feriştah Özçelik ${ }^{2}$, Tuğba Saraç3 \\ ${ }^{1}$ Eskişehir Osmangazi Üniversitesi, Mühendislik Mimarlık Fakültesi, Endüstri Mühendisliği Bölümü, Eskişehir, Türkiye (ORCID: 0000-0001-5363-3619) \\ ${ }^{2}$ Eskişehir Osmangazi Üniversitesi, Mühendislik Mimarlık Fakültesi, Endüstri Mühendisliği Bölümü, Eskişehir, Türkiye (ORCID: 0000-0003-0329-203X) \\ ${ }^{3}$ Eskişehir Osmangazi Üniversitesi, Mühendislik Mimarlık Fakültesi, Endüstri Mühendisliği Bölümü, Eskişehir, Türkiye (ORCID: 0000-0002-8115-3206)
}

(International Symposium on Multidisciplinary Studies and Innovative Technologies (ISMSIT) 2021 - 21-23 October 2021)

(DOI: 10.31590/ejosat.1017475)

\begin{abstract}
ATIF/REFERENCE: Ertem, M., Özçelik, F. \& Saraç, T. (2021). Stokastik İlişkisiz Paralel Makine Çizelgeleme Problemi için bir
\end{abstract} Matematiksel Model. Avrupa Bilim ve Teknoloji Dergisi, (29), 278-283.

$\ddot{O} \mathbf{z}$

Bir iş, aynı işlemi yapabilen makinaların herhangi birisinde işlem görebiliyor ise bu makinalar, paralel makinalar olarak adlandırılmaktadır. Eğer paralel makinaların bir iş için işlem süreleri farklılık gösteriyorsa bu makinalar iliş̧kisizdir. İlişkisiz paralel makine çizelgeleme problemi (UPM) hem endüstride yaygın bir uygulama alanına sahip olması hemde esnek atölye tipi çizelgeleme problemi gibi daha karmaşık problemlerin alt problemi olması nedeniyle çizelgeleme literatüründe önemli bir yere sahiptir. Problemin işlem süreleri, hazırlık süreleri, müşteri terminleri gibi parametrelerinin değerlerini kesin olarak belirlemek zordur. Bu parametreler taleplerin ve termin tarihlerinin müşteri tarafından son anda değiştirilmesi, makine arızaları, hammadde temininde aksamalar gibi pek çok faktöre bağlı olarak değişkenlik gösterebilmektedir. UPM doğası gereği stokastik bir yapıya sahiptir ve nedenle literatürde problemi deterministik olarak ele alan çok sayıda çalışmanın yanısıra stokastik olarak ele alan çalışmalar da mevcuttur. Ancak problemi stokastik olarak ele alan çalışmaların çoğu işlem sürelerinin stokastik olması durumunu incelemiş̧lerdir. Bu çalışmada ise literatürün genelinden farklı olarak sıra bağımlı hazırlık sürelerini stokastik olarak ele almanın katkısı araştıılımışır. Sıra bağımlı hazırlık süreli stokastik UPM için iki aşamalı stokastik programlama yaklaşımı kullanılmıştır. Stokastik problem için bir matematiksel model önerilmiştir. Önerilen modelin etkinliği rassal türetilen test problemleri üzerinde gösterilmiştir. Öncelikle tüm test problemleri parametrelerin deterministik olduğu varsayımı ile deterministik model ile çözülmüş ve çizelgeler elde edilmiştir. Daha sonra sıra bağımlı hazırlık süreleri stokastik olarak ele alınmış ve problemler önerilen stokastik model ile çözülmüştür. Son olarak her bir problem için sıra bağımlı hazırlık süresini stokastik ele almanın katkısı hesaplanmıştır. Yapılan testler, sadece 10 işin olduğu küçük boyutlu problemler için bile problemi stokastik olarak ele almanın son işin tamamlanma zamanını ortalama yüzde $\% 0,305$ kısalttığını ortaya koymuştur.

Anahtar Kelimeler: İlişkisiz parallel makina çizelgeleme problemi, İki aşamalı stokastik programlama, stokastik sıra bağımlı hazırlık süreleri.

\section{A Mathematical Model for Stochastic Unrelated Parallel Machine Scheduling Problem}

\begin{abstract}
If a job can be processed by any machines that can do the same operation, these machines are called parallel machines. If parallel machines have different processing times for a job, these machines are unrelated. The unrelated parallel machine scheduling problem (UPM) has an important place in the scheduling literature because it has a wide application area in the industry, and it is a sub-problem of more complex problems such as flexible job-shop scheduling problem. It is difficult to precisely determine the values of the parameters of the problem, such as processing times, setup times, due dates. These parameters may vary depending on many factors, such as changes in demands and due dates by the customer at the last moment, machine malfunctions, and disruptions in raw material supply. For this reason, UPM has a stochastic structure by nature, and there are many studies in the literature that deal with the problem as deterministic, as well as studies that deal with it as stochastic. However, most of the studies dealing with the problem as stochastic have examined the case of stochastic processing times. In this study, unlike the general literature, the contribution of handling the sequence-dependent setup times as stochastic was investigated. A two-stage stochastic programming approach is used for stochastic UPM with sequence-dependent setup time. A mathematical model is proposed for the stochastic problem. The effectiveness of the proposed model is demonstrated on randomly generated test problems. First of all, all test problems were solved with a deterministic model, assuming that the parameters were deterministic, and schedules were obtained. Then, the sequence-dependent setup times were
\end{abstract}

\footnotetext{
* Sorumlu Yazar: Eskişehir Osmangazi Üniversitesi, Mühendislik Mimarlık Fakültesi, Endüstri Mühendisliği Bölümü, Eskişehir, Türkiye, ORCID: 0000-0001-5363-3619, mertem@,ogu.edu.tr
} 
handled stochastic, and the problems were solved with the proposed stochastic model. Finally, the contribution of stochastic handling of the sequence-dependent setup time for each problem is calculated. Tests have revealed that even for small-sized problems with only 10 jobs, treating the problem as stochastic shortens the completion time of the last job by an average of $0.305 \%$.

Keywords: Unrelated parallel machine scheduling problem, Two-stage stochastic programming, stochastic sequence-dependent setup times.

\section{Giriş}

Çizelgeleme problemleri için ilk sistematik çözüm yaklaşımları 1950lerin ortasında önerilmiştir. O tarihten bu yana literatürde pek çok farklı üretim ortamını ele alan çok sayıda çalışma yapılmıştır. Makine çizelgeleme problemleri ile birçok farklı üretim ortamında karşılaşılmaktadır. Bu nedenle, üretim açısından hala önemli problemlerden biridir. Çizelgeleme problemleri makine ortamlarına göre tek makine, paralel makine, akış tipi ve atölye tipi çizelgeleme olmak üzere dört ana başlık altında incelenebilir. Paralel makine çizelgeleme problemleri aynı işi yapabilen makinaların çizelgelenmesi problemidir. Son yıllarda üretim sürelerinin farklılık gösterdiği ilişkisiz paralel makine çizelgeleme problemlerine olan ilgi artmıştır.

Makine çizelgeleme problemleri genellikle stokastik bir yapıya sahiptir. Bu nedenle literatürde problemi stokastik olarak ele alan birçok çalışma vardır. Literatürde yer alan stokastik makine çizelgeleme problemlerini ele alan çalışmaların çoğunluğunda işlem süreleri stokastiktir (Van den Akker and Hoogeveen, 2008; Soroush, 2010; Salmasnia et al., 2015; Chang et al., 2017; Atakan et al., 2017; Lemos and Ronconi, 2015; Gu and Lu, 2010; Iranpoor et al., 2013; Baker, 2014; Aydilek and Allahverdi, 2013).

Makine çizelgeleme çalışmalarının bazılarında hazır olma zamanları (Zhang et al., 2013), arızalar (Gu et al., 2014), sipariş geliş zamanları (Ronconi and Powell, 2010; Xu et al., 2015), hazırlık süreleri (Aydilek et al. 2013) ve sıra bağımlı hazırlık süreleri (Ertem et al., 2019) stokastik olarak ele alınmıștır. Ayrıca birden fazla parametreyi stokastik olarak ele alan çalışmalar da mevcuttur (Wang et al., 2015; Zhang et al., 2018; Allahverdi 2008; Allahverdi 2009; Aydilek et al. 2015; Sharma and Jain 2014).

Sıra bağımlı hazırlık süreli makine çizelgeleme problemiyle birçok farklı üretim ortamında karşılaşılmaktadır. Örneğin, plastik üretiminde renk değişimi, metal işlemede kalıp değişimi ve kağıt endüstrisinde kağıt bobinlerinin kesilmesi problemleri sıra bağımlı hazırlık süreleri gerektirirler. Bazı gerçek hayat problemlerinde, hazırlık süreleri hazırlık ekibinin yetenek düzeyleri, hazırlık sırasında kullanılan kaynakların durumu ve hazırlıkta kullanılacak araçların beklenmedik arızaları gibi nedenlerle stokastik olabilmektedir (Allahverdi, 2015). Allahverdi (2015), yaptığı kapsamlı literatür taramasında önemine rağmen sadece birkaç çalışmada stokastik hazırlık sürelerinin ele alındığını ve bu konuyu dikkate alan çalışmalara ihtiyaç olduğunu belirtmiştir. Stokastik parametreler birçok çizelgeleme probleminde yaygın olarak dikkate alınmış olmasına rağmen, sadece birkaç çalışmada sıra bağımlı hazırlık süreleri ile ilgili belirsizlik dikkate alınmaktadır.

Paralel makine çizelgeleme ile ilgili erişilebilen literatür dikkate alındığında, problemi stokastik olarak ele alan sadece bir çalışma vardır. Gu and Lu (2010) çalışmalarında özdeş paralel makine çizelgeleme problemini ele almışlardır. Stokastik kabul ettikleri parametre literatürün genelinde olduğu gibi 'işlem süreleri' dir. Ele aldıkları problemin amacı elde tutma maliyetinin enküçüklenmesidir. Bu çalışmada ise, $\mathrm{Gu}$ and $\mathrm{Lu}$ (2010)'dan farklı olarak özdeş paralel makine çizelgeleme probleminin genelleştirilmiş hali olan ilişkisiz paralel makine çizelgeleme problemi ele alınmıştır. Bu problemde bir işin işlem süresi her makinada farklılık gösterebilmektedir. Ayrıca yine ilgili çalışmada ele alınmamış olmasına rağmen bu çalışmada incelenen problemde bir işten diğerine geçişte hazırlık süreleri gerekli olup, bu süreler sıra ve makine bağımlı ve stokastiktir. $\mathrm{Bu}$ çalışmada $\mathrm{Gu}$ and $\mathrm{Lu}$ (2010)'dan farklı olarak, stokastik sıra bağımlı hazırlık sürelerinin son işin tamamlanma zamanına etkileri incelenmiştir.

\section{Materyal ve Metot}

\subsection{Deterministik Model}

$\mathrm{Bu}$ çalışmada motivasyon kaynağımız plastik enjeksiyon makinasının çizelgelenmesi problemidir. Plastik enjeksiyonla üretim, enjeksiyon makinesi ile gerçekleştirilen tek kademeli bir üretim şeklidir. Plastik enjeksiyonla üretim yapan firmalar genellikle küçük ve orta ölçekli yan sanayilerdir. Örneğin Eskişehir'de Arçelik buzdolabı fabrikasının yan sanayisi olarak çalışan ve plastik enjeksiyon ile üretim yapan çeşitli firmalar vardır. Bu tür işletmelerde karşılaşılan temel problemlerden birisi, siparişlerin eldeki makinelere yüklenmesi ve üretim sıralarının belirlenmesi problemidir. İşletmelerde genellikle birden çok enjeksiyon makinası olduğu için bu problem paralel makine çizelgeleme problemi olarak ele alınmaktadır. Makinaların farklı özelliklere sahip olması nedeniyle makineler ilişkisiz kabul edilmiştir.

Enjeksiyonla plastik parça üretiminde, koyu renkli bir parçadan sonra açık renkli bir parçanın üretimi çizelgelendiğinde ya da bazı özellikli hammaddelerin farklı yapıdaki hammaddelerden sonra kullanılması gerektiğinde, hammadde haznesinin tamamen temizlenmesi gerektiğinden hazırlık süreleri ciddi şekilde uzayabilmektedir. Öte yandan enjeksiyon makinelerinde bir ürünün üretilebilmesi için bu ürüne ait kalıbın ilgili makineye bağlanması gerekmektedir. Bir üründen diğerinin üretimine geçileceği zaman gerekecek hazırlık süresi, gerekiyorsa hammadde haznesinin temizlenmesi ve ilgili kalıbın makinaya bağlanması aşamalarından oluşur. $\mathrm{Bu}$ iki işlem paralel yapılabileceği için hangisi daha uzunsa, toplam hazırlık süresini o belirler. Ancak her iki hazırlık aşamasının da süresi bu işe ayrılabilecek iş gücü ve kalıp bağlamak için kullanılacak kreynin başka bir işlem için meşgul olup olmamasına ve makinanın tipine göre oldukça farklılık gösterebilmektedir. Eğer kreyn meşgulse, kalıp değişimi kreynin boş kalmasını beklemek zorundadır. Bu nedenle ele alınan problemde hazırlık süreleri çizelge sırasına ve makinaya bağlı ve stokastiktir.

Özetle, ele alınan problemde, $n$ iş, $m$ paralel makinede işlem görmektedir. $j$ işinin her makinedeki işlem süresi farklıdır. İşlerin hazırlık süreleri sıra ve makine bağımlıdır ve stokastiktir. İşlerin bölünmesine izin verilmemektedir ve işler sıfır zamanında hazırdır. Her makine aynı anda sadece bir iş gerçekleştirebilmektedir. Amaç fonksiyonu son işin tamamlanma zamanının enküçüklenmesidir.

Problemin 'sıra ve makine bağımlı hazırlık sürelerinin deterministik olduğu' varsayımıyla oluşturulan deterministik matematiksel model ve bu modele ait kümeler, indisler, parametreler, karar değişkenleri, kısıtlar ve amaç fonksiyonu aşağıda verilmiştir. 
Kümeler ve Indisler:

$\mathrm{N}=\{1,2, \ldots, n\}$ iş kümesi

$\mathrm{M}=\{1,2, \ldots, m\}$ makine kümesi

$i, j \in \mathrm{N}$ iş indisi

$k \in \mathrm{N}$ sira indisi

$l \in \mathrm{M}$ makine indisi

Parametreler:

$p_{j l}: j$. işin $l$. makinedeki işlem süresi $h_{j l}: j$. işin $l$. makinede ilk sıraya atanması durumunda hazırlık süresi

$s_{i j l}: i$ işi $j$ işinden önce $l$. makinede çizelgelenmişse sıra ve makine bağımlı hazırlık süresi

$M$ : yeterince büyük bir pozitif sayı

Karar Değişkenleri:

$x_{j k l}$ : eğer $j$. iş $k$. sırada $l$. makineye atandıysa 1 , diğer durumda 0 $C_{j}: j$ işinin tamamlanma zamanı

\section{Amaç fonksiyonu:}

enk $z=C_{\max }$

Kisitlar:

$$
\begin{array}{ll}
\sum_{j} x_{j k l} \leq 1 & \forall k, l \\
\sum_{k} \sum_{l} x_{j k l}=1 & \forall j \\
\sum_{j} x_{j k l}-\sum_{i} x_{i(k-1) l} \leq 0 & \forall k, l \quad k>1 \\
C_{j}+M\left(1-x_{j k l}\right) \geq h_{j l}+p_{j l} & \forall j, k, l \quad k=1 \\
C_{j}+M\left(2-x_{j k l}-x_{i(k-1) l}\right) \geq C_{i}+p_{j l}+s_{i j l} & \forall i, j, k, l \quad i \neq j, k>1 \\
C_{m a x} \geq C_{j} & \forall j \\
x_{j k l} \in\{0,1\} & \forall j, k, l \\
C_{j} \geq 0 & \forall j
\end{array}
$$

Amaç (1) son işin tamamlanma zamanının enküçüklenmesidir. Denklem (2-3) sirasıyla bir makinanın bir sırasına en fazla bir işin atanabilmesini ve her işin mutlaka bir makinanın bir sırasına atanmasııı sağlamaktadır. Denklem (4) aynı makineye atanan işlerin sıra atlamadan işlenmesini sağlamaktadır. Denklem (5) her makinedeki ilk işlerin ve denklem (6) ilk sıra haricindeki işlerin tamamlanma zamanını belirlemektedir. Denklem (7) son işin tamamlanma zamanını hesaplatma kısıtlarıdır. Denklem (89) karar değişkenlerin işaret kısıtlarıdır.
Problemin stokastik modeli ve gerekli ek küme, indisler ve parametreler aşağıda verilmiştir.

$W=\{1,2, \ldots, w\}$ senaryo kümesi $w \in W$ senaryo indisi $p r_{w}: w$. senaryonun gerçekleşme ihtimali $s_{i j l w}: i$ işi $j$ işinden önce $l$. makinede w. senaryoda çizelgelenmişse sıra ve makine bağımlı hazırlık süresi $C_{j w}: j$ işinin w. senaryodaki tamamlanma zamanı

\subsection{Stokastik Model}

Amaç fonksiyonu:

enk $z=\sum_{w} p r_{w} C_{\max }(w)$

Kisitlar:

$$
\begin{aligned}
& \sum_{j} x_{j k l} \leq 1 \\
& \sum_{k} \sum_{l} x_{j k l}=1
\end{aligned}
$$$$
\forall k, l
$$ 


$\begin{array}{ll}\sum_{j} x_{j k l}-\sum_{i} x_{i(k-1) l} \leq 0 & \forall k, l \quad k>1 \\ C_{j w}+M\left(1-x_{j k l}\right) \geq h_{j l}+p_{j l} & \forall j, k, l, w \quad k=1 \\ C_{j w}+M\left(2-x_{j k l}-x_{i(k-1) l}\right) \geq C_{i w}+p_{j l}+s_{i j l w} & \forall i, j, k, l, w \quad i \neq j, k>1 \\ C_{\text {max }}(w) \geq C_{j w} & \forall j, w \\ x_{j k l} \in\{0,1\} & \forall j, k, l \\ C_{j w} \geq 0 & \forall j\end{array}$

$\sum_{j} x_{j k l}-\sum_{i} x_{i(k-1) l} \leq 0$
Stokastik modelde amaç (10) son işin beklenen tamamlanma zamanının bütün senaryolar üzerinden enküçüklenmesidir. Diger denlemlerin hepsi deterministik modeldekiyle aynı kısitları ifade etmektedir, tek bir farkla ki Denklem 14, 15, 16 ve 18' de kstllar her bir senaryo için ayrı ayrı hesaba katılmaktadır.

\section{3. İki Aşamalı Stokastik Programlama}

Stokastik programlama (SP), değerleri belirsiz olan parametrelerin geçmiş veriler veya uzman görüşü kullanılarak değerlendirildiği optimizasyon problemlerinin modellenmesi için bir yaklaşımdır. Belirsiz parametreleri dikkate almanın bir başka yolu, verileri olasılık dağılımlarına dönüştürerek teorik dağılımlar oluşturmaktır. Düzeltme (recourse) modelleri, ilk aşama kararının belirsiz parametre değerlerinin gerçekleşmesinden önce alındığı en yaygın stokastik programlardır. Belirsiz parametrelerin bazıları gerçekleştikten sonra, önceki aşama kararında düzeltmeler yapılır ve ardından sonraki aşama kararı verilir. En yaygın kullanılan düzeltme modeli, ilk aşama kararının verildiği, ardından belirsizliğin gerçekleştiği ve ikinci aşamada düzeltme kararının verildiği iki aşamalı stokastik modeldir.

Deterministik yaklaşımda, tek bir değere ihtiyaç duyulduğundan geçmiş verileri kullanarak noktasal bir tahmin yapılır ya da bu verilerin ortalaması alınır. $\mathrm{Bu}$ yaklaşım, değişkenliği dikkate almaz ve sadece kullanılan tek değeri göz önünde bulundurarak çözüm türetir. Buna karşın, stokastik yaklaşım, parametrelerdeki değişkenliğin senaryolar aracıllığıyla hesaba katılmasına ve daha gerçekçi (değişkenliği öngören) bir çizelgeleme yapılmasına izin verir. Hazırlık sürelerindeki değişkenliğin senaryolaştırılabilmesi için geçmiş veriler bir dağılıma uydurularak (distribution fitting) Monte Carlo örnekleme metodu ile her senaryo için rastgele bir parametre değeri üretilir. Böylece, gerçekçi veriler kullanılarak yapılan çizelgeleme olası durumları hesaba katarak uzun dönemde daha başarılı sonuçlar sağlar. Stokastik yaklaşımı kullanmanın avantajını göstermek için deterministik ve stokastik yaklaşımları karşılaştırarak stokastik çözümün değeri (Value of Stochastic Solution-VSS) hesaplanabilir. VSS'yi hesaplama yönteminin adımları aşağıda verilmiştir.

Adım 1: Stokastik programlama problemi tüm senaryolar dikkate alınarak çözülür ve amaç fonksiyonu değeri $\left(\mathrm{z}_{\mathrm{s}}\right)$ hesaplanır.
Adım 2: Deterministik model kullanarak problem çözülür ve amaç fonksiyonunun değeri $\left(\mathrm{z}_{\mathrm{d}}\right)$ hesaplanır ve işlerin sırasını gösteren y vektörü belirlenir.

Adım 3: Adım 2'de bulunan iş sırasını (y vektörünü) sabitleyerek stokastik programlama probleminin amaç fonksiyonunun değeri tekrar ( $\left.\mathrm{Z}_{\mathrm{mv}}\right)$ hesaplanır.

Adım 4: VSS'yi hesaplamak için deterministik ve stokastik amaç fonksiyonu değerleri arasındaki fark (VSS $\left.=z_{m v}-Z_{s}\right)$ bulunur.

\section{Araştırma Sonuçları}

$\mathrm{Bu}$ çalışmada sıra bağımlı hazırlık sürelerinin stokastik olduğu UPM'nin çözümü için önerilen stokastik modelin performansını ölçebilmek için rassal olarak türetilmiş 10 işli test problemleri kullanılmıştır. Deterministik test problemi türetme yöntemimiz Rajendran ve Ziegler'in (2003) önerdiği yönteme benzemektedir. İşlem süreleri $\left(p_{j l}\right),[1,99]$ aralığında düzgün dağılıma uygun olarak türetilmiş̧tir. Sıra ve makine bağımlı hazırlık süreleri $\left(s_{i j l}\right),[1,99]$ ve $[51,149]$ aralığında iki farklı düzgün dağılımdan türetilmiştir. 10 iş 2 makine (10-2) için her bir problem grubundan 5'er adet örnek türetilmiştir. Test problemleri, sırasıyla iş-makina sayısı, sıra bağımlı hazırlık süresi türetme yöntemi ( $[1,99]$ aralığında ise $1,[51,149]$ aralığında ise 2$)$ ve örnek numarası dikkate alınarak kodlanmıştır. Örneğin, 10-2-21, 2 makinede işlem görecek 10 işin olduğu ve sıra bağımlı hazırlık sürelerinin $[51,149]$ aralığında türetildiği problemin ilk örneğini göstermektedir. Stokastik problem setleri, deterministik problem parametreleri kullanılarak oluşturulacaktır: stokastik sıra bağımlı hazırlık süreleri deterministik değerin \%10 ([0.90 $s_{i j l}^{d}$, $\left.\left.1.10 s_{i j l}^{d}\right]\right), \% 20\left(\left[0.80 s_{i j l}^{d}, 1.20 s_{i j l}^{d}\right]\right) \quad$ ve $\% 30\left(\left[0.70 s_{i j l}^{d}\right.\right.$, $\left.\left.1.30 s_{i j l}^{d}\right]\right)$ varyasyonu aralığında rastgele türetilmiştir. Toplamda 30 adet stokastik problem $(3 * 2 * 5)$ türetilmiştir.

Geliştirilen matematiksel modeller rassal türetilen test problemleri kullanılarak GAMS yazılımının CPLEX çözücüsü ile süre sınırı olmadan çözülmüştür. Tüm problemlerin eniyi çözümlerine ulaşılmıştır. Tablo-1'de elde edilen sonuçlar ve VSS değerleri verilmiştir. 
Tablo 1. Deterministik ve stokastik test problemlerinin amaç fonksiyon $\left(C_{\max }\right)$ değerleri, çözüm süreleri ve VSS değerleri

\begin{tabular}{|c|c|c|c|}
\hline & No & DETERN & İSTIK \\
\hline & No & $\mathrm{C}_{\max }$ (sa.) & Süre (s.) \\
\hline & $10-2-1-1$ & 268 & 240.45 \\
\hline & $10-2-1-2$ & 323 & 215.77 \\
\hline & $10-2-1-3$ & 310 & 246.02 \\
\hline & $10-2-1-4$ & 280 & 190.72 \\
\hline & $10-2-1-5$ & 314 & 196.14 \\
\hline $10 \%$ & $10-2-2-1$ & 524 & 984.34 \\
\hline & $10-2-2-2$ & 570 & 883.75 \\
\hline & $10-2-2-3$ & 583 & 1118.86 \\
\hline & $10-2-2-4$ & 507 & 636.95 \\
\hline & $10-2-2-5$ & 548 & 1262.91 \\
\hline & $10-2-1-1$ & 298 & 189.78 \\
\hline & $10-2-1-2$ & 283 & 148.33 \\
\hline & $10-2-1-3$ & 213 & 150.05 \\
\hline & $10-2-1-4$ & 262 & 183.41 \\
\hline $20 \%$ & $10-2-1-5$ & 247 & 156.19 \\
\hline $20 \%$ & $10-2-2-1$ & 551 & 1161.78 \\
\hline & $10-2-2-2$ & 498 & 873.88 \\
\hline & $10-2-2-3$ & 502 & 881.36 \\
\hline & $10-2-2-4$ & 517 & 626.13 \\
\hline & $10-2-2-5$ & 538 & 33801.64 \\
\hline & $10-2-1-1$ & 228 & 115.89 \\
\hline & $10-2-1-2$ & 314 & 281.06 \\
\hline & $10-2-1-3$ & 286 & 177.97 \\
\hline & $10-2-1-4$ & 256 & 130.19 \\
\hline 300 & $10-2-1-5$ & 273 & 206.98 \\
\hline $30 \%$ & $10-2-2-1$ & 516 & 857.42 \\
\hline & $10-2-2-2$ & 557 & 987.35 \\
\hline & $10-2-2-3$ & 563 & 1451.96 \\
\hline & $10-2-2-4$ & 517 & 904.17 \\
\hline & $10-2-2-5$ & 571 & 726.83 \\
\hline
\end{tabular}

\begin{tabular}{|c|c|c|}
\hline \multicolumn{2}{|c|}{ STOKASTİK } & \multirow{2}{*}{ VSS } \\
\hline $\mathrm{C}_{\max }$ (sa.) & Süre (s.) & \\
\hline 268.23 & 24956.41 & 0 \\
\hline 322.83 & 24864.63 & $\mathbf{0}$ \\
\hline 309.77 & 123037.81 & 0.13 \\
\hline 279.77 & 25270.81 & 0.10 \\
\hline 314.03 & 27201.31 & $\mathbf{0}$ \\
\hline 526.80 & 108456.39 & 1.13 \\
\hline 569.60 & 113658.20 & 0 \\
\hline 583.30 & 136934.25 & 3.97 \\
\hline 509.10 & 79905.25 & 0 \\
\hline 547.13 & 176714.33 & 0.83 \\
\hline 299.80 & 38638.22 & 0 \\
\hline 282.40 & 20685.39 & $\mathbf{0}$ \\
\hline 214.10 & 49434.70 & $\mathbf{0}$ \\
\hline 261.70 & 27655.63 & $\mathbf{0}$ \\
\hline 246.27 & 18464.45 & $\mathbf{0}$ \\
\hline 556.80 & 210277.02 & $\overline{\mathbf{0}}$ \\
\hline 504.30 & 213968.19 & $\mathbf{0}$ \\
\hline 499.00 & 112639.84 & 4 \\
\hline 522.67 & 213219.31 & $\mathbf{0}$ \\
\hline 537.93 & 199945.44 & 3.7 \\
\hline 228.20 & 15582.95 & 3.10 \\
\hline 314.27 & 39529.69 & $\mathbf{0}$ \\
\hline 287.17 & 25410.84 & $\mathbf{0}$ \\
\hline 256.93 & 12357.89 & $\mathbf{0}$ \\
\hline 272.03 & 25065.83 & 2.67 \\
\hline 522.97 & 210369.35 & $\overline{\mathbf{0}}$ \\
\hline 550.60 & 154325.21 & 2.57 \\
\hline 563.43 & 187985.27 & 16.17 \\
\hline 520.87 & 98429.88 & 5.17 \\
\hline 581.60 & 131604.66 & $\mathbf{0}$ \\
\hline
\end{tabular}

Tablo 1'den de görülebileceği gibi 10 iş iki makine olan küçük problemlerde bile 30 problemin 12'sinde pozitif VSS elde

\section{Sonuç}

$\mathrm{Bu}$ çalışmada sıra bağımlı hazırlık sürelerinin stokastik olduğu UPM ele alınmıştır. Ele alınan problemin çözümü için iki aşamalı stokastik programlama yaklaşımı kullanılmıştır. $\mathrm{Bu}$ yaklaşım gereği çözülmesi gereken deterministik ve stokastik UPM için matematiksel modeller kullanılmıştır. Problemin deterministik versiyonu için literatürde matematiksel modeller mevcuttur. Ancak stokastik model bu çalışma kapsamında önerilmiştir. Her iki modelinde eniyi çözümlerinin elde edilebilmesi için rassal olarak türetilmiş 10 işli test problemleri kullanılmıştır. Yapılan testler, sıra bağımlı hazırlık sürelerinin stokastik olduğu durumlarda bu parametreyi deterministik kabul etmenin son işin tamamlanma zamanını $\% 0,315$ uzattığını ortaya koymuştur. Daha çok işin olduğu büyük problemlerde bu kaybın katlanarak büyüyeceği öngörülmektedir. Ancak problem boyutu büyüdükçe özellikle stokastik modelle problemi çözmek çok süre gerektirebilir veya bir uygun çözüme ulaşmak bile mümkün olamayabilir. Bu nedenle gelecek çalışmalarda deterministik ve stokastik UPM problemlerinin bir metasezgisel algoritma ile çözüldüğü iki aşamalı stokastik programlama yaklaşımları kullanılarak, sıra bağımlı hazırlık sürelerini stokastik ele almanın büyük boyutlu problemlere katkısı incelenebilir. edilmiştir. Bu sonuç sıra bağımlı hazırlık sürelerini stokastik olarak ele almanın katkısını açıkça ortaya koymaktadır.

\section{Teşekkür}

$\mathrm{Bu}$ çalışma, TÜBITTAK tarafindan 120M886 nolu proje kapsamında desteklenmiştir.

\section{Kaynakça}

Allahverdi, A. 2008. "Three-machine flowshop scheduling problem to minimize makespan with bounded setup and processing times." Journal of the Chinese Institute of Industrial Engineers 25(1): 52-61.

Allahverdi, A. 2009. "Three-machine flowshop scheduling problem to minimize maximum lateness with bounded setup and processing times." Journal of Operations and Logistics 2: $1-11$.

Allahverdi, A. 2015. "The third comprehensive survey on scheduling problems with setup times/costs." European Journal of Operational Research 246: 345-378.

Atakan, S. , K. Bülbül · and N. Noyan. 2017. "Minimizing valueat-risk in single-machine scheduling" Ann Oper Res 248:2573.

Aydilek, A., H. Aydilek, and A. Allahverdi. 2013. "Increasing the profitability and competitiveness in a production environment with random and bounded setup times. "International Journal of Production Research 51: 106-117.

Aydilek, H., A. Aydilek, and A. Allahverdi. 2015. "Production in a two-machine flowshop scheduling environment with 
uncertain processing and setup times to minimize makespan.” International Journal of Production Research 53: 2803-2819.

Aydilek, H., and A. Allahverdi. 2013. "A polynomial time heuristic for the two-machine flowshop scheduling problem with setup times and random processing times." Applied Mathematical Modelling 37 (12-13): 7164-7173.

Baker, K.R., 2014, "Minimizing earliness and tardiness costs in stochastic scheduling", European Journal of Operational Research, 236, 445-452.

Chang, Z., S. Song, Y. Zhang, J.Y. Ding, R. Zhang, and R. Chiong. 2017. "Distributionally robust single machine scheduling with risk aversion." European Journal of Operational Research 256: 261-274.

Ertem M., Ozcelik F., Sarac T. (2019), "Single machine scheduling problem with stochastic sequence-dependent setup times", International Journal of Production Research, DOI: $10.1080 / 00207543.2019 .1581383$.

Gu, J., M. Gu, and X. Gu. 2014. "Optimal Rules for Single Machine Scheduling with Stochastic Breakdowns." Mathematical Problems in Engineering 1-9.

Gu, M. and Lu, X., 2010, "Stochastic scheduling problem with varying weight for each job", ASIA-PACIFIC JOURNAL OF CHEMICAL ENGINEERING, 5, 681-689.

Iranpoor, M., Fatemi Ghomi, S.M.T., Zandieh, M., 2013, "Duedate assignment and machine scheduling in a low machinerate situation with stochastic processing times" Computers \& OperationsResearch, 40, 1100-1108.

Lemos, R.F., Ronconi, D.P. 2015. "Heuristics for the stochastic single-machine problem with $\mathrm{E} / \mathrm{T}$ costs". International Journal of Production Economics 168:131-142.

Rajendran, C., \& Ziegler, H. 2003. "Scheduling to minimize the sum of weighted flowtime and weighted tardiness of jobs in a flowshop with sequence-dependent setup times." European Journal of Operational Research, 149(3), 513-522.

Ronconi, D.P., and W.B. Powell. 2010. "Minimizing total tardiness in a stochastic single machine scheduling problem using approximate dynamic programming." Journal of Scheduling 13: 597-607.

Salmasnia, A., M. Khatami, R.B. Kazemzadeh, S.H. Zegordi. 2015. "Bi-objective single machine scheduling problem with stochastic processing times." TOP, 23:275-297.

Sharma,P., and A. Jain. 2014. "Analysis of dispatching rules in a stochastic dynamic jobshop manufacturing system with sequence-dependent setup times." Frontiers of Mechanical Engineering 9(4): 380-389.

Soroush, H.M. 2010. "Solving a stochastic single machine problem with initial idle time and quadratic objective." Computers \& Operations Research 37: 1328-1347.

Van den Akker, M., and H. Hoogeveen. 2008. "Minimizing the number of late jobs in a stochastic setting using a chance constraint." Journal of Scheduling 11: 59-69.

Wang, D.J., Liu, F., Wang, Y.Z. and Jin, Y., 2015. “A knowledgebased evolutionary proactive scheduling approach in the presence of machine breakdown and deterioration effect." Knowledge-Based Systems, 90, pp.70-80.

$\mathrm{Xu}$, L., Wang, Q. and Huang, S., 2015. "Dynamic order acceptance and scheduling problem with sequence-dependent setup time." International Journal of Production Research, 53(19), pp.5797-5808.

Zhang L., Y. Lin, Y. Xiao and X. Zhang. 2018. "Stochastic singlemachine scheduling with random resource arrival times." International Journal of Machine Learning and Cybernetics 9(7): 1101-1107.
Zhang, J., Yang, W., Tu, Y. 2013, "Scheduling with compressible and stochastic release dates", Computers \&OperationsResearch, 40, 1758-1765. 